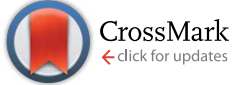

Cite this: RSC Adv., 2017, 7, 1936

Received 20th October 2016 Accepted 22nd November 2016

DOI: 10.1039/c6ra25508f

www.rsc.org/advances

\section{Formulation of concentrated and stable ink of silver nanowires with applications in transparent conductive films $\uparrow$}

\begin{abstract}
Chao Chen, ${ }^{a b}$ Yonggao Jia, ${ }^{a b}$ Dan Jia, $^{\text {ab }}$ Shuxin $\mathrm{Li}^{\text {a }}$ Shulin $\mathrm{Ji}^{* a}$ and Changhui Ye ${ }^{* a}$
Silver nanowire transparent conductive films have been proposed as a promising candidate to replace indium tin oxide films for electronic applications. Stable ink with a high loading amount of silver nanowires is essential for the scale-up of the production of transparent conductive films considering the storage need due to separated procedures of nanowire synthesis and film formation. It is very hard to re-disperse the nanowires if irreversible aggregation happens during storage for coating. The paradox of stabilizing the concentrated nanowire ink and facile utilizing of the ink for highly conductive films with a low junction resistance at the joint points of organic stabilizer-capped silver nanowires has to be solved. A two-step method has been provided to overcome the difficulty in this work. First, a silver nanowire ink with the loading amount of $5 \mathrm{mg} \mathrm{mL}^{-1}$ is formulated, which is stable for more than three months under normal conditions. The success in maintaining the long-term stability lies in applying an appropriate amount of surfactant and choosing organic solvents with appropriate mixing ratios to strengthen the interaction of the surfactant with the silver nanowires and to decrease the free energy through enthalpy of mixing silver nanowires into the solvent for aggregation prevention. Second, the concentrated ink at any time is simply washed a suitable number of times and diluted to a uniform dispersion for coating of TCFs with excellent optical and electrical performance, having a figure of merit $\sigma_{\mathrm{DC}} / \sigma_{\mathrm{OP}}$ (ratio of the electrical conductance to optical conductance) as high as 228 . The viability of treating silver nanowires in large quantity paves the way for real industrial applications.
\end{abstract}

\section{Introduction}

In the past decades, silver nanowires (AgNWs) have drawn tremendous attention due to their promising applications in flexible electronic devices by forming transparent conductive films (TCFs). ${ }^{1-7}$ The performance of TCFs containing AgNWs is comparable to the state-of-the-art indium tin oxide (ITO) films, while with additional merits, such as low production cost, being indium free and flexible against mechanical touching or bending. Usually, AgNWs are dispersed in ethanol after synthesis as a dispersion for direct coating on a substrate. The dispersion is not stable for long-term storage and if irreversible aggregation happens during storage, it is very hard to redisperse the nanowires for coating and non-uniform TCFs could be anticipated. To improve the stability of AgNW

${ }^{a}$ Anhui Key Laboratory of Nanomaterials and Technology, Key Laboratory of Materials Physics, Institute of Solid State Physics, Chinese Academy of Sciences, Hefei 230031, China. E-mail: slji@issp.ac.cn; chye@issp.ac.cn; Fax: +86-551-65591434; Tel: +86551-65591923

${ }^{b}$ University of Science and Technology of China, Hefei 230026, China

$\dagger$ Electronic supplementary information (ESI) available: Photographs and viscosity change with time of AgNW inks, SEM images and TGA curves of AgNWs, and a table of the residual PVP content of AgNW inks. See DOI: $10.1039 / \mathrm{c} 6 \mathrm{ra} 25508 \mathrm{f}$ dispersion, organic additives should be employed to prepare AgNW ink for long-term storage and transportation. For this purpose, it is required to increase the loading amount of AgNWs in the ink, and before use, it could be diluted to uniform dispersion with a desired concentration for coating, whose value depends on the coating methods. However, recipe for concentrated and long-term stable AgNW ink is still lacking.

Many researchers have demonstrated AgNW dispersions with good stability at a low concentration for a short term. For example, Jin et al. implemented a binder of poly(dopamine) and alginic acid to acquire a $0.65 \mathrm{mg} \mathrm{mL}^{-1} \mathrm{AgNW}$ ink. ${ }^{8} \mathrm{Li}$ et al. obtained a $5 \mathrm{wt} \%$ AgNW ink in ultra pure water, ${ }^{9}$ and $\mathrm{Hu}$ et al. prepared a $2.7 \mathrm{mg} \mathrm{mL}{ }^{-1} \mathrm{AgNW}$ ink in methanol. ${ }^{10}$ Reported AgNW dispersions with a high concentration usually suffer from aggregation under a long-term storage condition. For example, Tao et al. prepared a $15 \mathrm{wt} \%$ AgNW ink using only alcohols by mixing under ultra-sonication, and precipitation could be anticipated after a certain period. ${ }^{11} \mathrm{Hu}$ et al. introduced polyethylene oxide (PEO) as a dispersing agent to obtain a $9.6 \mathrm{mg} \mathrm{mL} \mathrm{m}^{-1} \mathrm{AgNW}$ ink by vigorous stirring; however as pointed out by the authors, PEO would precipitate together with AgNWs at room temperature to generate solid composite sediments. ${ }^{12} \mathrm{Wu}$ et al. formulated a 10-20 wt\% AgNW ink under the assistance of a double amino-silane capping agent; yet original 
PEDOT:PSS ink was based to formulate the PEDOT:PSS and AgNWs hybrid ink, which could hamper the neutral color of coated films. ${ }^{13}$ These inks could be used to coat TCFs on a particular substrate; however, the long-term stability of these inks poses severe problems for transportation, storage, and utilization. Therefore, to make AgNW TCFs viable in real-world applications, the formulation of stable AgNW inks with a high concentration is essential.

The difficulty in preparing stable ink with a high loading amount of AgNWs lies in that the ink should be both stable and ready to utilize. To improve the stability of the ink, one could add organic stabilizers that could not only increase the viscosity of the ink but also suppress the aggregation of AgNWs. However, the organic stabilizers will inevitably reduce the conductivity of TCFs because the junction resistance will be much too high for organic molecule-stabilized AgNWs. ${ }^{14}$ Therefore, the organic stabilizers should be readily removable before coating TCFs. In this paper, the paradox was solved by a two-step method: first, $5 \mathrm{mg} \mathrm{mL}{ }^{-1} \mathrm{AgNW}$ ink based on ethylene glycol (EG), $N$-methyl pyrrolidone (NMP), glycerol and poly(vinylpyrrolidone) (PVP) was formulated, which can be stable at room temperature for more than 3 months; second, the concentrated ink was simply washed and diluted to uniform dispersion for coating of TCFs with high performance. The reason why choosing these organic solvents and additives for ink formation are as follows. Mixed solvents based on NMP have been explored to effectively enhance the stability of the pristine single-walled carbon nanotubes (SWCNT) dispersion through tuning solubility parameters of final solvent, which has been theoretically analyzed in quantity combining DLVO theory, Flory-Huggins theory and Hildebrand/Hansen solubility parameters. ${ }^{15}$ Besides NMP, organic solvents of EG and others have been identified to form graphene oxide (GO) dispersions with long-term stability, ${ }^{16}$ probably due to large dipole moment (3.75 for NMP and 2.31 for EG) and suitable Hildebrand/Hansen solubility parameter. ${ }^{17}$ EG has also been employed in flexible electronics as the humectant in stabilizing the ink of AgNWs. ${ }^{18}$ Traditionally, glycerol acts both as viscosity modifier and wetting agent in preparing directly writing inks with a relative long-term stability. ${ }^{19,20}$ PVP was chosen to make use of its steric hindrance arising from the long-chain for stability improvement, ${ }^{21}$ and NMP along with PVP has been proven an efficient system in improving the stability of graphite inks than the pure NMP dispersions..$^{22,23}$

Herein, the relative content of NMP to EG in our ink was systematically investigated along with the concentration of PVP, based on the consideration of tuning Hildebrand/Hansen solubility parameters of final dispersing medium for AgNWs, including the Hansen hydrogen bonding parameter $\left(\delta_{\mathrm{H}}\right)$ arising from hydroxyl and the Hansen polar parameter $\left(\delta_{\mathrm{P}}\right)$ originating from carbonyl. ${ }^{15}$ The content of glycerol was fixed at $10 \mathrm{vol} \%$ because of the more tough dispersive capacity depending on its viscosity as high as $1412 \mathrm{mpa}$ s. TCFs of high performance could be coated by using the ink after a brief cleaning to partially remove organic stabilizers on the surface of AgNWs.

\section{Experimental section}

\subsection{Materials}

$\mathrm{AgNO}_{3}(\geq 99.8 \%$, Shanghai Qiangshun Chemical Reagent Co., Ltd) was used as a source material. Ethylene glycol (EG, Sinopharm Chemical Reagent Co., Ltd) was used as a reductant in a synthetic process. Poly(vinylpyrrolidone) (PVP) (average molecular weight (MW) of 55 000, PVP-55000) and PVP (average MW of 360000 , PVP-360000) (Sigma-Aldrich) were used as capping agents during the growth of AgNWs. N,N-Dimethylformamide (DMF, Sinopharm Chemical Reagent Co., Ltd) was used as a chemical agent to purify AgNW ink for TCF formation. Glycerol and $N$-methyl pyrrolidone (NMP) (Sinopharm Chemical Reagent Co., Ltd) were used in the recipe for ink formulation. PVP (average MW of 1300 000, PVP-1300000) (Aladdin Industrial Corporation) was explored as both a viscosity modifier and a stabilizer in the ink. All reagents were used as-received without further purification.

\subsection{Preparation of AgNWs and stable ink}

AgNWs were synthesized by reducing $\mathrm{AgNO}_{3}$ in the presence of PVP in EG as reported in our early work with minor modifications. ${ }^{21}$ Briefly, a mixture of $0.0812 \mathrm{~g}$ PVP-360000 and $0.0842 \mathrm{~g}$ PVP- 55000 was dissolved into $22 \mathrm{~mL}$ of EG. Subsequently, 2.5 $\mathrm{mL}$ of $\mathrm{FeCl}_{3}$ solution $(600 \mu \mathrm{M}$ in $\mathrm{EG})$ and $\mathrm{AgNO}_{3}$ solution $(0.180 \mathrm{~g}$ in $3 \mathrm{~mL}$ of EG) were added rapidly into PVP solution within $1 \mathrm{~min}$. The synthesis was carried out at $130{ }^{\circ} \mathrm{C}$ for $130 \mathrm{~min}$. After cooling to room temperature, the product was washed with $30 \mathrm{~mL}$ de-ionized (DI) water, centrifuged, and collected as the source for the preparation of concentrated AgNW ink. In a typical procedure for the preparation of AgNW ink, $1.5 \mathrm{~g}$ PVP-1300000 was dissolved in a mixture of $20 \mathrm{~mL}$ NMP, $25 \mathrm{~mL}$ EG, and $5 \mathrm{~mL}$ glycerol under a vigorous stirring for $5 \mathrm{~h}$. Then $6 \mathrm{~mL}$ of the mixture was applied to disperse $30 \mathrm{mg}$ AgNWs with a homogeneous stirring for $30 \mathrm{~min}$ at room temperature. Finally, AgNW ink with a concentration of $\sim 5 \mathrm{mg}$ $\mathrm{mL}^{-1}$ was prepared.

\subsection{Fabrication of TCFs using AgNW ink}

The as-prepared AgNW ink is stable up to several months under normal storing conditions. Before the coating of TCFs, AgNW ink was dispersed in DMF (or DI water), followed by centrifugation to partially remove PVP, and then re-dispersed in ethanol with a final concentration of $0.4 \mathrm{mg} \mathrm{mL}^{-1}$. TCFs were coated by doctor blading without any post-treatment.

\subsection{Characterizations}

The morphology of AgNWs was characterized by scanning electron microscopy (SEM) (Sirion 200 FEG) and transmission electron microscopy (TEM) (JEM-2010). Photographs were captured using an optical microscope equipped with a digital camera (SOIF-55XA). Thermo gravimetric analysis (TGA) was performed using a Pyris-1 analyzer (PerkinElmer). The viscosity measurements were made on a viscometer (NDJ-5S) at $25{ }^{\circ} \mathrm{C}$. Sheet resistance of TCFs was measured using a four-point probe 
resistivity meter (RTS-9). Optical transmittance spectra were obtained on a UV-Vis-NIR spectrometer (Shimadzu SolidSpec3600). FT-IR measurements were performed using a Nexus FTIR spectrometer (Thermo Nicolet NEXUS FT-IR spectrometer). Raman spectra were recorded on a Micro-RAMAN Spectroscopy (Renishaw inVia Reflex, LAICA DM2500, $\lambda=532 \mathrm{~nm}$ ).

\section{Results and discussion}

A modified polyol method as reported in our previous work was employed to synthesize AgNWs with average length up to $72 \mu \mathrm{m}$ and average diameter of $36 \mathrm{~nm}$ (ESI: Fig. S1†). In an initial stage, Raman measurements were carried out to investigate the effect of the volume ratio of NMP to EG $\left(V_{\mathrm{NMP}}: V_{\mathrm{EG}}\right)$ on the stability of inks with different formulations when the concentration of PVP, AgNWs and glycerol were fixed at $7.5 \mathrm{mg} \mathrm{mL}^{-1}, 0.3 \mathrm{mg}$ $\mathrm{mL}^{-1}$ and 10 vol\%, respectively. As shown in Fig. 1, two representative peaks centered at $\sim 213 \mathrm{~cm}^{-1}$ and $\sim 240 \mathrm{~cm}^{-1}$ were observed for the system whatever the solvent ratio was. The former one could be assigned to $\mathrm{Ag}-\mathrm{O}$ stretching vibration in $\mathrm{AgO}$, and the latter one is originated from $\mathrm{Ag}-\mathrm{O}$ stretching vibration between the carbonyl and AgNWs. ${ }^{24-28}$ The former peak has nearly a constant center while the latter one changes its center with the solvent ratio. We found that at a volume ratio of $4: 5$, the frequency of stretching vibration between the $\mathrm{O}$ in carbonyl and Ag in AgNWs is the largest, indicating the strongest force constant $(k)$ between silver and oxygen atoms due to the most sufficient overlapping of electron clouds between the carbonyl $(>\mathrm{C}=\mathrm{O})$ in PVP and AgNWs.$^{29}$ As a consequence, the ink is most stable, which was substantiated by the observation of the stability of the inks (ESI: Fig. S2 $\dagger$ ).

The mechanism on the stability of AgNW dispersion relating with the volume ratio of NMP to EG could be explained by the competition between the viscosity tuning by EG and the dipoledipole intermolecular force strengthening from carbonyls in NMP and PVP. Density of electron clouds surrounding PVP was elevated when NMP was employed as an additional additive, which could enhance the intermolecular force between carbonyl species and AgNWs as shown in Fig. 1a. In addition, embedded NMP molecules in the interval between PVP molecules was expected to provide additional space repulsion potential energy to stabilize AgNW dispersion from irreversible aggregation. Moreover, varying the volume ratio of NMP to EG changed the Hildebrand/Hansen solubility parameters of final solvent, which tuned the free energy through enthalpy of mixing AgNWs into the solvent; minimum energy could be achieved at a proper ratio when the Hildebrand/Hansen parameter of solvent equaled to that of AgNWs to formulate the most stable ink. ${ }^{15}$

However, when the concentration of AgNWs ( $\left.C_{\mathrm{AgNWs}}\right)$ increased from 0.3 up to $1.2 \mathrm{mg} \mathrm{mL}^{-1}$, the ink became less stable because PVP in the ink was not sufficient to stabilize AgNWs from aggregation (ESI: Fig. S3†).$^{10}$ When we simultaneously increased the concentration of PVP $\left(C_{\mathrm{PVP}}\right)$ from 7.5 to $30 \mathrm{mg} \mathrm{mL}^{-1}$ and fixing the solvent ratio at the optimal value, the ink became stable again. Fig. 2 indicated that the stretching vibration between $\mathrm{Ag}$ in AgNWs and oxygen atom in carbonyl shifted up to $244 \mathrm{~cm}^{-1}$ after adopting $30 \mathrm{mg} \mathrm{mL}^{-1}$ of $C_{\mathrm{PVP}}$. However, further increasing PVP (a)

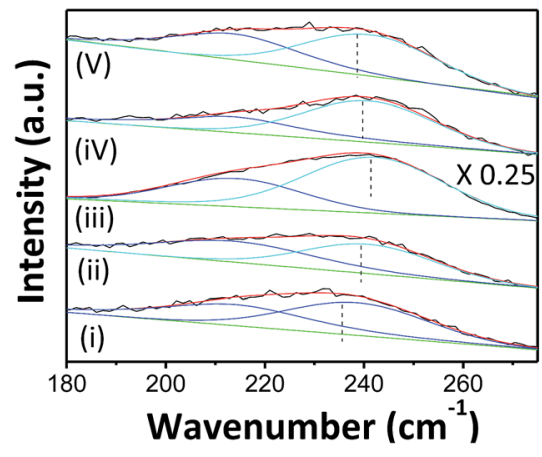

(b)

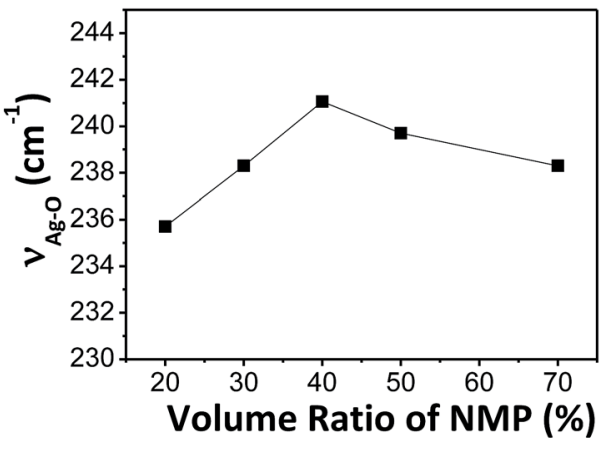

(c)

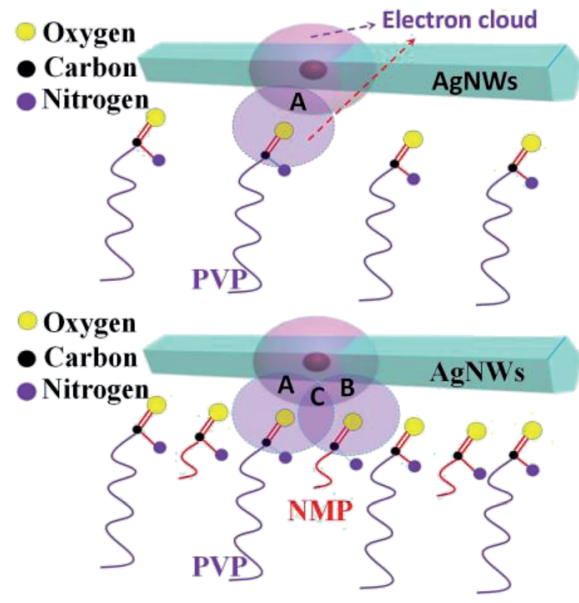

Fig. 1 (a) Raman spectra of AgNW inks stored for two weeks, which contained NMP, EG and glycerol with volume ratio of (i) $2: 7: 1$, (ii) $3: 6: 1$, (iii) $4: 5: 1$, (iv) $5: 4: 1$, and (v) $7: 2: 1$, while $C_{\mathrm{PVP}}, C_{\mathrm{AgNWs}}$, and the volume ratio of glycerol in the solvent $\left(C_{\text {glycerol }}\right)$ were kept as $7.5 \mathrm{mg} \mathrm{mL}^{-1}, 0.3 \mathrm{mg} \mathrm{mL}^{-1}$ and 10 vol\%; (b) Raman peak position for $\mathrm{Ag}-\mathrm{O}$ stretching vibration between the carbonyl and AgNWs obtained from (a); (c) schematic diagram depicting the mechanism of NMP stabilizing PVP-capped AgNW dispersion.

concentration led to the aggregation of AgNWs in the ink, possibly due to the decrease of solubility (ESI: Fig. S4†). Finally, a concentrated AgNW ink of $\sim 5 \mathrm{mg} \mathrm{mL}^{-1}$ was formulated where $C_{\mathrm{PVP}}$ and $V_{\mathrm{NMP}}: V_{\mathrm{EG}}: V_{\text {glycerol were fixed at } 30 \mathrm{mg} \mathrm{mL}} \mathrm{mL}^{-1}$ and $4: 5: 1$, respectively.

The resultant $5 \mathrm{mg} \mathrm{mL}^{-1}$ AgNW ink prepared with the optimal recipe was characterized using TEM. Fig. 3a and b display photographs and the SEM image of the AgNW ink, where no apparent sediment or non-uniformity could be observed revealing good dispersion for long-term stability. An 
(a)

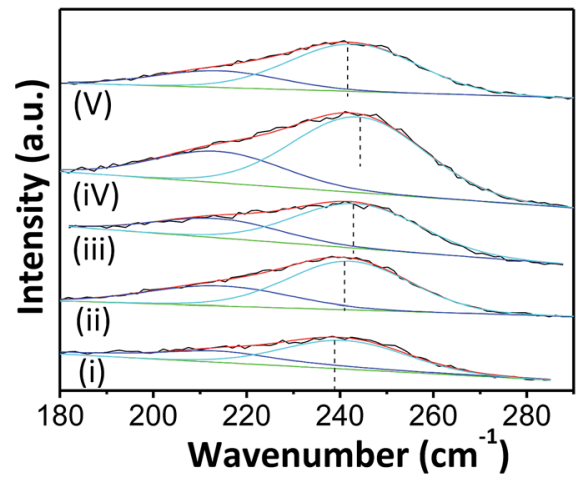

(b)

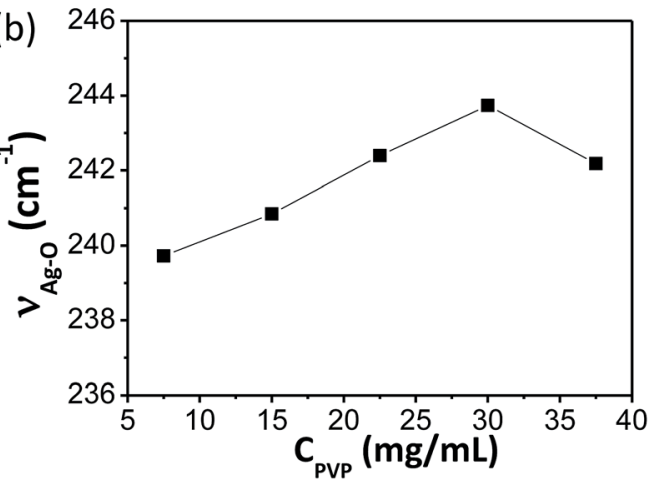

Fig. 2 (a) Raman spectra of AgNW inks stored for two weeks, sampled from suspensions where $C_{P V P}$ is (i) 7.5, (ii) 15, (iii) 22.5, (iv) 30 and (v) $37.5 \mathrm{mg} \mathrm{mL}{ }^{-1}$, while $V_{\mathrm{NMP}} / V_{\mathrm{EG}} / V_{\text {glycerol }}$ and $C_{\mathrm{AgNW}}$ were kept as $4: 5: 1$ and $1.2 \mathrm{mg} \mathrm{mL}^{-1}$; (b) Raman peak position for $\mathrm{Ag}-\mathrm{O}$ stretching vibration obtained from (a).

amorphous layer with a thickness of $\sim 5 \mathrm{~nm}$ was detected on the surface of AgNWs, probably corresponding to the thickness of the adsorbed PVP layer (Fig. 3c). Moreover, AgNW ink produced according to this recipe exhibits a long-term stability up to 6 months (ESI: Fig. S5†).

Fig. 4 displays the infrared spectra of pure PVP and PVPencapsulated AgNW ink. FT-IR spectrometry has been proven a powerful technique to monitor the interaction between PVP and metal nanoparticles. ${ }^{30-35}$ From curve (a), peak positioned at $1662 \mathrm{~cm}^{-1}$ should arise from the fundamental $\mathrm{C}=\mathrm{O}$ stretching vibration since its characteristic peak was supposed to be located at this region. ${ }^{36} \mathrm{~A}$ red-shift of $12 \mathrm{~cm}^{-1}$ could be detected for carbonyl when a vigorous stirring was applied to dissolve AgNWs homogeneously into the PVP dispersion, indicating the attachment of PVP to the surface of AgNWs through the interaction of the lone pair electrons of oxygen in PVP and the vacant orbitals of Ag. ${ }^{29}$

Viscosity tests at set intervals were carried out to verify the stability of $5 \mathrm{mg} \mathrm{mL}{ }^{-1} \mathrm{AgNW}$ ink as shown in Fig. S6. $\dagger$ No obvious change of the viscosity had been observed, indicating the stability of the ink for at least 3 months.

The capability to formulate such concentrated AgNW ink with long-term stability enables the storage of AgNWs against irreversible aggregation. It will be shown in the following paragraph that the ink could be used to prepare high-quality TCFs after gentle cleaning to partially remove organics on the (a)

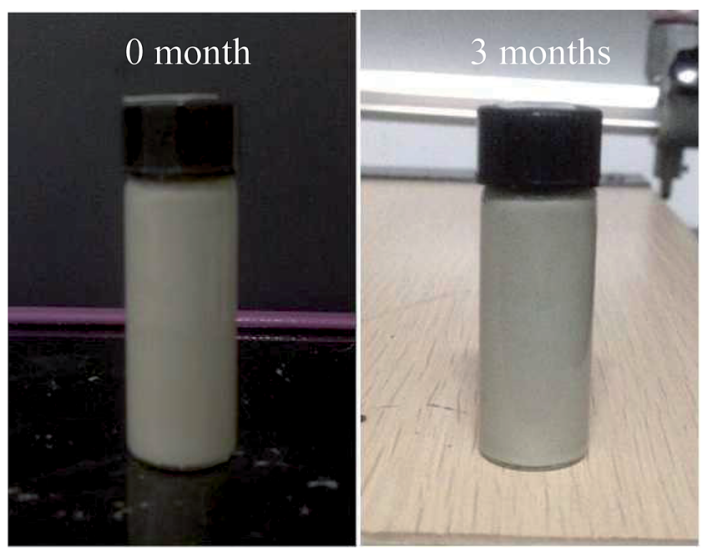

(b)

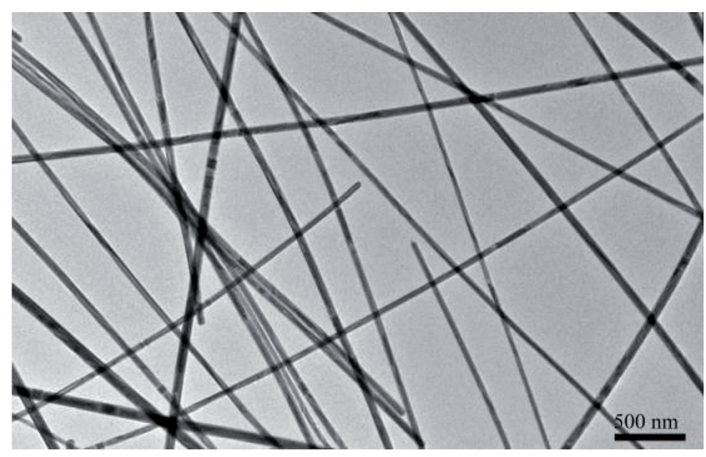

(c)

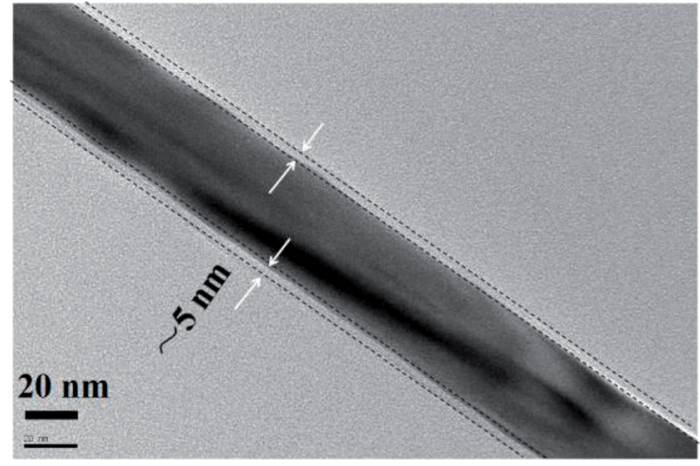

Fig. 3 (a) Photographs of the $5 \mathrm{mg} \mathrm{mL}^{-1}$ AgNW ink freshly made and stored for 3 months; (b) low and (c) high magnification TEM images of the ink stored for 3 months.

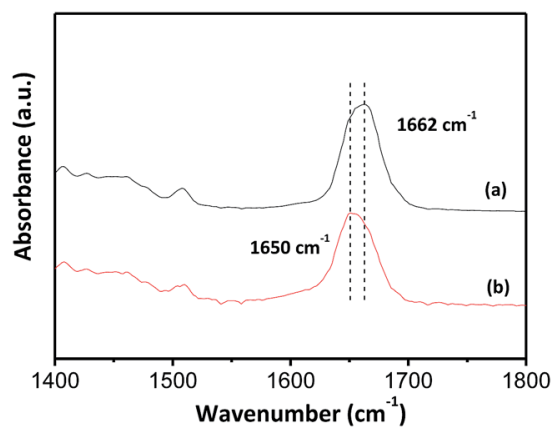

Fig. 4 FT-IR spectra of (a) pure PVP dissolved in a mixture of NMP, EG, and glycerol, and (b) $5 \mathrm{mg} \mathrm{mL}^{-1}$ PVP-encapsulated AgNW ink. 
surface of AgNWs before coating films. DI water and DMF were employed as treating solvents, respectively. TGA was used to detect the residual PVP on the surface of the AgNWs since the decomposition temperature for PVP was estimated as $300{ }^{\circ} \mathrm{C}^{32,37}$ Fig. S7 in ESI $\dagger$ and 5 show evidence that PVP could be removed by the two solvents efficiently since the relative weight of PVP on the surface of AgNWs exhibited a decrease from $30 \%$ to $2.7 \%$ when 1 cycle cleaning was applied.

After gentle cleaning, the purified AgNWs were coated on PET substrates. Fig. $6 a$ and b show that $T \%$ above $90 \%(\lambda=550$ $\mathrm{nm}$ ) and and $R_{\mathrm{s}}$ below $50 \Omega$ could be achieved. From Fig. $6 \mathrm{c}$ and $\mathrm{d}$, it is observed that 2 cleaning cycles by DMF generated optimum overall performance of AgNW TCFs. The figure of merit $\varphi_{\mathrm{TE}}$ is defined by Haacke as ${ }^{38}$

$$
\varphi_{\mathrm{TE}}=\frac{T^{10}}{R_{\mathrm{s}}}
$$

where $T$ is the transmittance and $R_{\mathrm{S}}$ is the sheet resistance. Recently, another figure of merit has also been widely utilized and defined as the ratio of the electrical conductance to optical conductance $\left(\sigma_{\mathrm{DC}} / \sigma_{\mathrm{OP}}\right)^{39,40}$

$$
T(\lambda)=\left(1+\frac{188.5}{R_{\mathrm{s}}} \frac{\sigma_{\mathrm{OP}}}{\sigma_{\mathrm{DC}}}\right)^{-2}
$$

where $\sigma_{\mathrm{DC}}$ and $\sigma_{\mathrm{OP}}$ are the DC conductivity and the optical conductivity at $550 \mathrm{~nm}$, respectively. From Table 1, conductive film coated with AgNWs purified with DMF for 2 cycles shows a value of $\sigma_{\mathrm{DC}} / \sigma_{\mathrm{OP}}$ as high as 228 at a relative lower surface density (ESI: Fig. S7†), which is superior to the recently reported values.
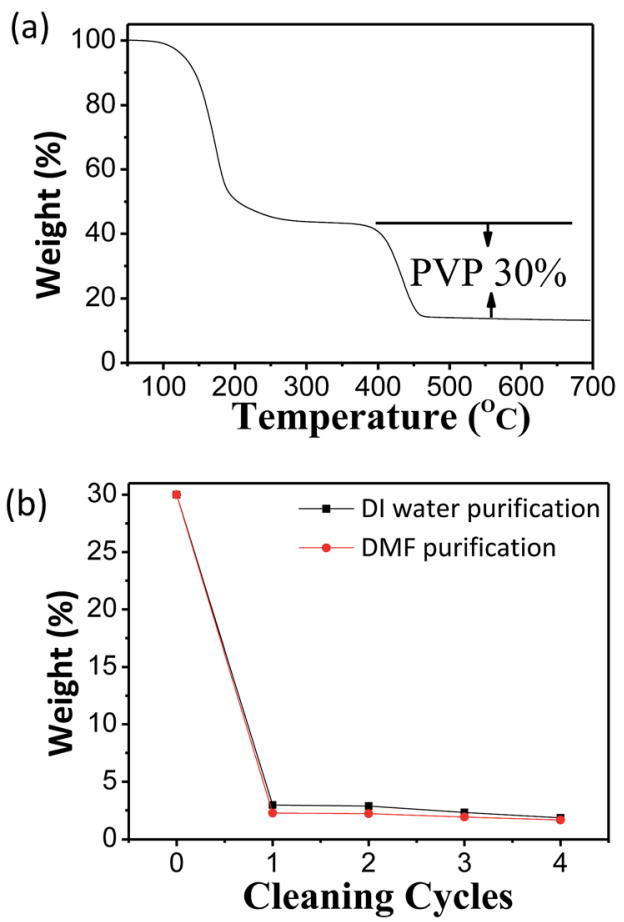

Fig. 5 (a) TGA curve of AgNW ink dried for $24 \mathrm{~h}$ in the vacuum oven at $80^{\circ} \mathrm{C}$. (b) The weight of residual PVP in the purified AgNWs versus cleaning cycles with DI water and DMF, respectively. (a)

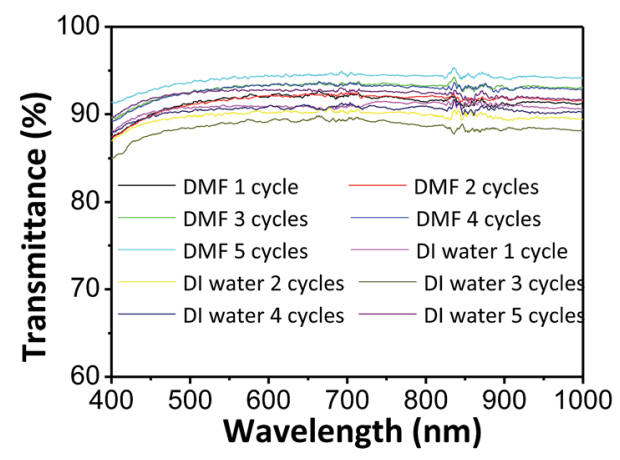

(b)

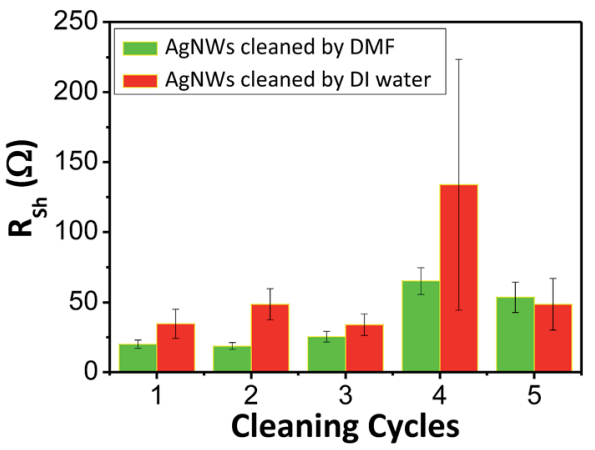

(c)

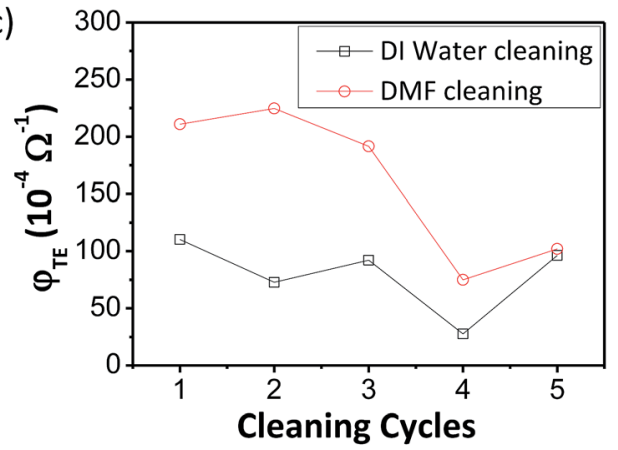

(d)

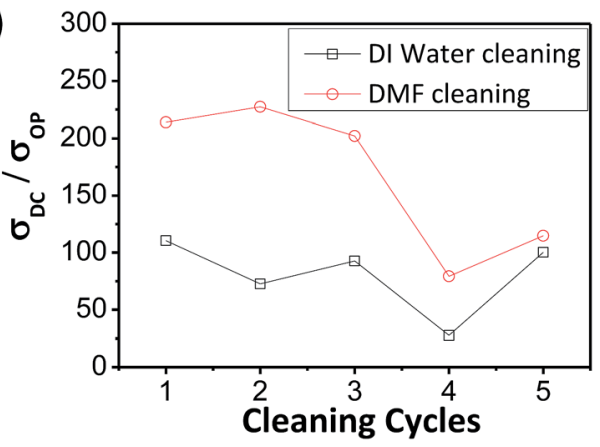

Fig. 6 Comparison of (a) transmittance (T\%), (b) sheet resistance $\left(R_{\mathrm{sh}}\right)$, (c) figure of merit $\left(\varphi_{\mathrm{TE}}\right)$, and (d) $\sigma_{\mathrm{DC}} / \sigma_{\mathrm{OP}}(\lambda=550 \mathrm{~nm})$ of films coated on PET by AgNWs cleaned for various cycles with DMF and DI water, respectively.

The transparent conductive films coated by AgNWs cleaned with DMF for 2 cycles exhibit relatively high comprehensive performance due to (i) the AgNWs with a large aspect ratio as high as 2000, (ii) less organics on the surface of AgNWs, and (iii) 
Table 1 Comparison of optical and electrical performance between our optimal transparent conductive films coated by AgNWs purified with DMF for 2 cycles and those in reported work

\begin{tabular}{llllll}
\hline $\begin{array}{l}M / A \\
\left(\mathrm{mg} \mathrm{m}^{-2}\right)\end{array}$ & $\begin{array}{l}R_{\mathrm{sh}} \\
(\Omega \text { per square })\end{array}$ & $T \%$ & $\begin{array}{l}\varphi_{\mathrm{TE}} \\
\left(10^{-4} \Omega^{-1}\right)\end{array}$ & $\sigma_{\mathrm{DC}} / \sigma_{\mathrm{OP}}$ & Ref. \\
\hline 32 & 182 & 88 & 15.3 & 15.7 & 41 \\
93 & 110 & 92 & 39.5 & 40.3 & 10 \\
28 & 13 & 85 & 151 & 171 & 39 \\
60 & 18.7 & 91.7 & 225 & 228 & This \\
& & & & & work \\
& & & & & \\
\hline
\end{tabular}

a much lower surface density. More importantly, the performance of the films was tested directly after coating without further physical or chemical treatment as done by others, which dramatically simplifies the production process of AgNW transparent conductive films.

\section{Conclusions}

In summary, we have formulated concentrated AgNW inks with good stability against aggregation by adjusting the content of organic additives. The ink could be applied to coat highperformance AgNW transparent conductive films after a brief cleaning of AgNWs prior to the coating process. It is noteworthy that the films exhibit a large figure of merit without post treatment after coating. The ability to produce concentrated AgNW inks with good stability and ease of utilization will bring this material even closer to industrial applications.

\section{Acknowledgements}

This work was supported by National Natural Science Foundation of China (Grant No. 11274308, 51501182, 51502295 and U1532268), and the CAS/SAFEA International Partnership Program for Creative Research Teams.

\section{Notes and references}

1 N. Komoda, M. Nogi, K. Suganuma and K. Otsuka, ACS Appl. Mater. Interfaces, 2012, 4, 5732.

2 S. J. Lee, Y.-H. Kim, J. K. Kim, H. Baik, J. H. Park, J. Lee, J. Nam, J. H. Park, T.-W. Lee, G.-R. Yi and J. H. Cho, Nanoscale, 2014, 6, 11828.

3 H. G. Cheong, R. E. Triambulo, G. H. Lee, I. S. Yi and J. W. Park, ACS Appl. Mater. Interfaces, 2014, 6, 7846.

4 N. Komoda, M. Nogi, K. Suganuma, K. Kohno, Y. Akiyama and K. Otsuka, Nanoscale, 2012, 4, 3148.

5 M. Layani, A. Kamyshny and S. Magdassi, Nanoscale, 2014, 6, 5581.

6 G. W. Huang, H. M. Xiao and S. Y. Fu, Nanoscale, 2014, 6, 8495.

7 S. L. Ji, W. W. He, K. Wang, Y. X. Ran and C. H. Ye, Small, 2014, 10, 4951.

8 Y. Jin, D. Deng, Y. Cheng, L. Kong and F. Xiao, Nanoscale, 2014, 6, 4812.
9 R. Z. Li, A. Hu, T. Zhang and K. D. Oakes, ACS Appl. Mater. Interfaces, 2014, 6, 21721.

10 L. Hu, H. S. Kim, J.-Y. Lee, P. Peumans and Y. Cui, ACS Nano, 2010, 4, 2955.

11 Y. Tao, Y. X. Tao, L. Y. Wang, B. B. Wang, Z. G. Yang and Y. L. Tai, Nanoscale Res. Lett., 2013, 8, 147.

12 M. Hu, J. Gao, Y. Dong, K. Li, G. Shan, S. Yang and R. K.-Y. Li, Langmuir, 2012, 28, 7101.

13 L. Y. L. Wu, W. T. Kerk and C. C. Wong, Thin Solid Films, 2013, 544, 427.

14 J. Wang, J. Jiu, T. Araki, M. Nogi, T. Sugahara, S. Nagao, H. Koga, P. He and K. Suganuma, Nano-Micro Lett., 2015, 7,51 .

15 M. W. Forney and J. C. Poler, J. Phys. Chem. C, 2011, 115, 10531.

16 J. I. Paredes, S. Villar-Rodil, A. Martínez-Alonso and J. M. D. Tascón, Langmuir, 2008, 24, 10560.

17 D. Konios, M. M. Stylianakis, E. Stratakis and E. Kymakis, J. Colloid Interface Sci., 2014, 430, 108.

18 N. Komoda, M. Nogi, K. Suganuma, K. Kohno and Y. Akiyama, Nanoscale, 2012, 4, 3148.

19 H.-T. Wang, O. A. Nafday, J. R. Haaheim, E. Tevaarwerk, N. A. Amro, R. G. Sanedrin, C.-Y. Chang, F. Ren and S. J. Pearton, Appl. Phys. Lett., 2008, 93, 143105.

20 B. Y. Ahn, D. J. Lorang and J. A. Lewis, Nanoscale, 2011, 3, 2700.

21 Y. Ran, W. He, K. Wang, S. Ji and C. Ye, Chem. Commun., 2014, 50, 14877.

22 E. Ou, Y. Xie, C. Peng, Y. Song, H. Peng, Y. Xiong and W. Xu, RSC Adv., 2013, 3, 9490.

23 T. Hasan, V. Scardaci, P. H. Tan, A. G. Rozhin, W. I. Milne and A. C. Ferrari, J. Phys. Chem. C, 2007, 111, 12594.

24 G. I. N. Waterhouse, G. A. Bowmaker and J. B. Metson, Phys. Chem. Chem. Phys., 2001, 3, 3838.

25 D. Büchel, C. Mihalcea, T. Fukaya, N. Atoda and J. Tominaga, MRS Online Proc. Libr., 2001, 674, V3.2.

26 I. Martina, R. Wiesinger, D. Jembrih-Simbuerger and M. Schreiner, e-Preserv. Sci., 2012, 9, 1.

27 N. R. C. Raju, K. J. Kumar and A. Subrahmanyam, J. Phys. D: Appl. Phys., 2009, 42, 135411.

28 H. Mao, J. Feng, X. Ma, C. Wu and X. Zhao, J. Nanopart. Res., 2012, 14, 887.

29 Z. T. Zhang, B. Zhao and L. M. Hu, J. Solid State Chem., 1996, 121, 105.

30 Y. Borodko, S. E. Habas, M. Koebel, P. Yang, H. Frei and G. A. Somorjai, J. Phys. Chem. B, 2006, 110, 23052.

31 C.-J. Lee, M. R. Karim, T. Vasudevan, H.-J. Kim, K. Raushan, M.-J. Jung, D.-Y. Kim and M.-S. Lee, Bull. Korean Chem. Soc., 2010, 31, 1993.

32 Y. Borodko, H. S. Lee, S. H. Joo, Y. Zhang and G. Somorjai, J. Phys. Chem. C, 2010, 114, 1117.

33 M. Behera and S. Ram, Int. Nano Lett., 2013, 3, 1.

34 W.-X. Tu, X.-B. Zuo and H.-F. Liu, Chin. J. Polym. Sci., 2008, 26, 23.

35 X. Lu, M. Niu, R. Qiao and M. Gao, J. Phys. Chem. B, 2008, 112, 14390.

36 D. P. McDermott, J. Phys. Chem., 1986, 90, 2569. 
37 Y. K. Du, P. Yang, Z. G. Mou, N. P. Hua and L. Jiang, J. Appl. Polym. Sci., 2006, 99, 23.

38 G. Haacke, J. Appl. Phys., 1976, 47, 4086.

39 S. De, T. M. Higgins, P. E. Lyons, E. M. Doherty, P. N. Nirmalraj, W. J. Blau, J. J. Boland and J. N. Coleman, ACS Nano, 2009, 3, 1767.
40 J. van de Groep, P. Spinelli and A. Polman, Nano Lett., 2012, 12, 3138.

41 X. Y. Zeng, Q. K. Zhang, R. M. Yu and C. Z. Lu, Adv. Mater., 2010, 22, 4484. 